\title{
JASP for Audit: Bayesian Tools for the Auditing Practice
}

\begin{abstract}
Statistical theory is fundamental to many auditing guidelines and procedures. In order to assist auditors with the required statistical analyses, and to advocate state-of-the-art Bayesian methods, we introduce JASP for Audit (JfA). JfA is easy-to-use, free-of-charge software that automatically follows the standard audit workflow, selects the appropriate statistical analysis, interprets the results, and produces a readable report. This approach reduces the potential for statistical errors and therefore increases audit quality. Next to the frequentist methods that currently dominate audit practice, JfA incorporates Bayesian counterparts of these methods that come with several advantages. For example, Bayesian statistics allows incorporation of expert knowledge directly into the statistical analyses, allowing for a decrease in sample size, and an increase in efficiency. In sum, JfA is designed with the auditor in mind, it guides the auditor through the statistical aspects of an audit, and therefore has the potential to increase audit efficiency and quality.
\end{abstract}

Keywords: audit software, Bayesian statistics, financial auditing, JfA.

\section{INTRODUCTION}

In many countries, listed organizations are required to be audited by law. In the United States alone there were 4,397 listed companies that had at least one audit performed in 2017 (TheWorldBank 2019). Listed companies are the greatest consumers of audit services worldwide, but also several private companies, nonprofit organizations, and governments are required to obtain audits. An audit is often complex, quality requirements are high, and the associated costs are substantial, on average between $\$ 87,000$ and $\$ 337,000$ (De George, Ferguson, and Spear 2012; Niemi 2005; Webb and Waymire 2016). We developed JASP for Audit (JfA) to facilitate the statistical aspects of an audit, it has the potential to decrease audit complexity while at the same time improve understanding of audit theory and, therefore, audit quality (Weidenmier and Herron 2004).

The reason why financial audits are required by law in many countries is that they evaluate the extent to which an organization's financial statements are presented fairly. It is the auditor's task to gather appropriate and sufficient evidence to determine whether the 
organization's statements contain errors that surpass a specified percentage or monetary amount, the so-called materiality. When the total error surpasses the materiality it is said that the financial statements contain material misstatement, which means that the errors in the financial statements may impact decisions of someone relying on those statements (IFAC 2010a). In a nutshell, a financial audit is a final check before an organization releases its yearly numbers to inform stakeholders of the organization about its financial situation.

From a data perspective, the financial statements of an organization are large populations of observations that can contain millions of transactions (Neter and James 1975; Ramage, Krieger, and Spero 1979; Titera 2013). As a result, manually evaluating the entire population is a time consuming and expensive task. It is therefore common practice for auditors to evaluate a subset of the organization's financial statements and — based on this subset - infer the total amount of misstatement in the population (Elder, Akresh, Glover, Higgs, and Liljegren 2013; IFAC 2010e). It is crucial that the inference is made carefully, as the auditor's judgment will influence the opinion of stakeholders and investors of the organization. Since the auditor's opinion is a sensitive one, the use of formal statistical techniques to make inferences has become standard practice in the field in order to substantiate the auditor's judgment (AICPA 2017).

With the current developments in data analytics (e.g., machine learning, big data), audit organizations are increasingly exposed to the opportunities of using data-driven methods and automated processes (for examples, see Alles, Kogan, and Vasarhelyi 2008; Appelbaum, Kogan, and Vasarhelyi 2017; Cao, Chychyla, and Stewart 2015; Durney, Elder, and Glover 2014; Earley 2015; Kogan 2019; McAfee, Brynjolfsson, Davenport, Patil, and Barton 2012; Titera 2013). Even though these developments provide auditors with opportunities to perform full population testing (Brown-Liburd et al. 2015), the fact remains that the use of statistical sampling in problematic accounts is still, and will be for a while, 
standard practice in financial audits (Bedingfield 1975; Christensen, Elder, and Glover 2015; Glover, Taylor, and Wu 2017; Hitzig 1995). Consequently, today's auditor must be qualified in multiple areas of statistical inference, which is not necessarily part of an auditor's core expertise (Dzuranin, Jones, and Olvera 2018; Ramachandran Rackliffe and Ragland 2016; Sledgianowski, Gomaa, and Tan 2017).

Hence, problems that arise during the statistical aspects of an audit are often due insufficient knowledge of statistical theory, complex software implementing statistical theory, and finally interpreting the resulting statistics correctly. Firstly, the auditor must have sufficient knowledge of the statistical theory underlying the audit procedures. For example, Beasley, Carcello, and Hermanson (2001) found that in 36 percent of their analyzed audit reports, the auditor failed to gather sufficient statistical evidence to back up their statement. Moreover, many auditors are not able to effectively incorporate statistical audit analyses in their work and therefore only use it on an ad-hoc basis (Li, Dai, Gershberg, and Vasarhelyi 2018). Secondly, the auditor must have access to software that is easy-to-use and performs analyses according to the International Standards on Auditing (IFAC 2010c). For instance, Nieschwietz, Pany, and Zhang (2002) and Weidenmier and Herron (2004) argue that teaching students to work with audit software packages was useful. However, software packages like IDEA, ACL, and TeamMate Analytics are closed source (Caseware Analytics 2018; Galvanize 2018; Wolters Kluwers 2018). They have steep licensing costs and offer no access to their source code, making it unclear how results are obtained. Thirdly, the auditor must be able to interpret the results of said analyses correctly, while it is known that the misinterpretation of statistics is not uncommon in fields where statistics is prevalent (Héroux 2016; Wasserstein and Lazar 2016, Johnstone 1997, Morey, Hoekstra, Rouder, Lee, and Wagenmakers 2016). 
To help overcome the statistical hurdles that can occur during an audit, the International Federation of Accountants offers guidelines in the form of the International Standards on Auditing (ISA). These guidelines specify what the auditor should and should not do and help the auditor in setting up the design of their audit (AICPA 2017). Extending the suggestion from Brown-Liburd et al. (2015), we argue that integrating these guidelines with existing statistical software has the potential to increase audit efficiency and quality; and therefore we have developed a software package that makes it exceptionally easy for auditors to adhere to these guidelines.

For the abovementioned reasons, we developed JASP for Audit (JfA), a module within the statistical software package JASP ${ }^{1}$ (JASP Team 2018; Love, Selker, Marsman, Jamil, Dropmann, Verhagen, Ly, Gronau, Smira, Epskamp et al. 2018) that is built specifically to help the auditor perform their statistical analyses and interpret the results correctly. An important benefit of JfA is grounded in the fact that auditors are not necessarily educated in statistics and might find statistical inference a challenging subject. To assist auditors with the statistical aspects of an audit, JfA uses the ISA guidelines to guide them through the audit workflow based on the type of data and audit question and automatically selects the appropriate corresponding statistical technique. Furthermore, JfA generates an audit report containing the results and the statistical interpretation of these results (preventing common misinterpretations and unjustified inferences). This approach minimizes the dependency on an auditor's statistical knowledge so that any auditor can use JfA, while increasing their understanding of the statistical theory underlying the audit process. Note that JASP and JfA are released under an open-source license, which means both will always be available to the audit community free of charge. Open-source software has many other

\footnotetext{
${ }^{1}$ JASP and JfA can be downloaded from the JASP website (https://jasp-stats.org/).
} 
advantages over closed-source packages (AlMarzouq, Zheng, Rong, and Grover 2005; Maxwell 2006), namely that it offers access to its source code and can be easily transformed or extended further by anyone with the required knowledge.

From a statistical perspective JfA offers the commonly used frequentist analyses, but it also provides the auditor with state-of-the-art Bayesian techniques which were previously not readily available in an audit context. Benefits of Bayesian statistics for the auditor are discussed later in this article, but in general one may argue that Bayesian estimation can increase audit quality by incorporating prior knowledge from the auditor in a statistically correct manner (Kinney 1983, Stewart 2013).

To illustrate JfA's approach to statistical auditing, we will introduce the reader to the standard audit sampling workflow (hereafter: "audit workflow") in section two. This workflow can be separated into four stages, each with a specific purpose. In section three we will discuss the statistical elements in this workflow, and show where Bayesian statistics can improve upon frequentist methods. Section four will show the structure of JfA, and how it facilitates the auditor in their workflow. As an illustration, a monetary walkthrough of the Bayesian audit workflow in JfA will be discussed. The last section presents our concluding comments.

\section{THE AUDIT WORKFLOW: A PRACTICAL PERSPECTIVE}

The audit workflow follows a traditional design of estimating an unknown population parameter, which in the context of financial auditing means the estimated amount of misstatement (AICPA 2017; Burgstahler and Jiambalvo 1986; Schwartz 1997). In order to do so, auditors have a four-stage approach; planning the sample, selecting the corresponding 
sample from the population, executing the substantive tests, and evaluating the subset to draw inferences about the population (Felix and Kinney 1982).

To illustrate the audit workflow we consider the case of BuildIt, a fictional company in the United States that sells building materials. BuildIt is being audited by Laura, an external auditor for a fictional audit firm. Suppose that Laura strives to state that, with 95 percent certainty, no more than five percent of the organization's total balance contains an error. In BuildIt's balance of approximately $\$ 1.4$ million, that five percent corresponds to $\$ 70,161$. Errors are defined as misstatements in the financial statements and occur when the recorded transactions do not match the organization's true incomes and expenses (IFAC 2010d). In order for Laura to be able to draw a statistical conclusion about BuildIt's financial statements, she follows the audit workflow.

\section{Stage 1: Planning}

In the planning stage the auditor assesses relevant information that is gathered during earlier stages of the audit to determine an appropriate sample size. Note that information is relevant for the auditor if it can be used to adjust the amount of audit evidence required to assess the quality of the financial statements (for example, information about the quality of the organization's internal control systems), thereby directly impacting the number of transactions that they need to evaluate.

In the case of BuildIt, Laura starts by calculating the number of units from the total balance that she needs to audit, given her expectations about the number of errors in the population. She calculates a sample size such that, when the expected errors are at most 2.5 percent in the sample (she may base this percentage on last year's audit where she observed at most 2.5 percent errors), the population can be approved with 95 percent confidence. For instance, Laura's calculations show that she needs to select 169 units and audit their 
corresponding transactions to gather support for the claim that, with 95 percent confidence, the misstatement is lower than the materiality of five percent.

\section{Stage 2: Selection}

The auditor uses the resulting sample size from the planning stage as input for the selection stage. Here, the auditor has to determine what the so-called sampling units are. In statistical selection all sampling units in the population receive an inclusion probability, and are selected with that probability until the required sample size has been reached.

The most commonly used sampling method for the audit procedure is monetary unit sampling (MUS) (Christensen, Elder, and Glover 2015; Swanepoel 2018). In MUS, probabilities are assigned on the level of individual monetary units (e.g., a dollar). When a dollar is selected to be in the sample, the transaction that includes that dollar is selected. This favors higher transactions, as a transaction of $\$ 5,000$ is five times more likely to be selected than a transaction of $\$ 1,000$. Another often used sampling method is called record sampling, a method where probabilities are assigned on the transaction level, treating transactions with a high value and a low value the same, a transaction of $\$ 5,000$ is equally likely to be selected as a transaction of $\$ 1,000$. Note that there may also be occasions in which records do not represent transactions (e.g., university diplomas). Here, only record sampling would apply, as the monetary value of the diplomas is not the subject of the audit. In the remainder of this article we will use the term transactions, even if records do not strictly spoken represent transactions.

In the case of BuildIt, Laura uses MUS to select 169 transactions proportional to their book values.

\section{Stage 3: Execution}


In this stage, the auditor will check whether the transactions are correct. The most straightforward annotation method considers the transactions to be either correct or incorrect. This method does not consider the magnitude of the misstatement in individual transactions, and results in a fairly conservative estimate of the total misstatement. A more common method takes the true size of the transaction (the audit value) into account. Here, information about the size of the misstatement proportional to the size of the transaction is taken into account, resulting in a more accurate and less conservative estimate of the total misstatement (Fienberg, Neter, and Leitch 1977). The monetary nature of the audit population determines the manner of annotation. If the auditor wishes to make a statement on the value of the misstatement, it is common practice to annotate their selection with their audit values.

In the case of BuildIt, Laura will assess the audit value of the 169 selected transactions and annotate the selection with their corresponding audit values, since she wants to make a statement on the value of the misstatement.

\section{Stage 4: Evaluation}

In the evaluation stage the auditor uses the annotated sample from the execution stage to make a well-substantiated inference about the total population misstatement. To this aim, the auditor applies a statistical method to calculate the estimated maximum misstatement in the population (e.g., an $x$-percent confidence bound), and approves the population when this estimate is below the materiality.

In the case of BuildIt, Laura evaluates her annotated sample with the appropriate statistical method, yielding a 95 percent confidence bound on the misstatement in BuildIt's financial statements. Laura's estimate of the maximum misstatement is 2.77 percent (or $\$ 38,802$ ), which is lower than materiality (five percent or $\$ 70,161$ ). Therefore, she can 
conclude that, with 95 percent confidence, BuildIt's financial statements do not contain material misstatements.

\section{THE AUDIT WORKFLOW: A STATISTICAL PERSPECTIVE}

Considering the size of audit populations, it would be enormously expensive to make an audit assertion with absolute certainty. Since the auditor cannot evaluate the total population of financial statements but wants to make a population statement with a certain amount of confidence, statistical inference is a prerequisite. Recognizing this, the auditor defines a probability that they will provide an incorrect opinion on the population of financial statements, the audit risk. To quantify the audit risk in probabilistic terms, the International Standards on Auditing consider the audit risk model (ARM).

In this section we will explain the rationale behind the ARM, and discuss the link between the statistical elements of the ARM and the audit workflow. Furthermore, we discuss a Bayesian method for incorporating those statistical elements in an audit, and provide an applied example of this philosophy.

\section{The Audit Risk Model}

The ARM provides a mathematical association between the specified audit risk and the assessed risk of material misstatement (Knechel 2007). According to the ARM, the audit risk is divided into three constituents; inherent risk, control risk, and detection risk. Inherent risk is the risk of a material misstatement due to an error in a financial statement before consideration of any related controls. Control risk is the risk of a material misstatement not prevented or detected by the internal control systems of the organization (e.g., computer managed databases). Detection risk is the risk that an auditor will fail to find material misstatements that exist in an organization's financial statements. 
While detection risk is a probability statement, inherent risk and control risk are defined by the ISA and are commonly assessed by the auditor on a three-point scale consisting of the categories low, medium, and high risk. In order to calculate the audit risk (see Equation 1), these categories need to be mapped onto probabilities according to audit firm standards. An example of such a mapping is 50 percent, 60 percent, and 100 percent for low, medium, high risk respectively as proposed by the Dutch government (IODAD 2007). For a given level of audit risk, the tolerable level of detection risk bears an inverse relationship to the two assessed risks of material misstatement, see Equation 1. Intuitively, a lower risk of material misstatement may require a higher tolerable detection risk and, accordingly, requires less persuasive audit evidence (IFAC 2010a).

\section{Audit risk $=$ Inherent risk $\times$ Control risk $\times$ Detection risk}

The ARM is practically useful because it enables the auditor to incorporate prior knowledge on the control environment to increase or decrease the required amount of audit evidence. To illustrate the functioning of the ARM, having found that the control risk of an organization is medium (e.g., 60 percent) the auditor may increase the detection risk from the original $\alpha_{0}=0.05$, or 5 percent to $\alpha_{1}=\frac{0.05}{1 \times 0.60}=0.0833$, or 8.33 percent. When both inherent risk and control risk are judged as high (100 percent), the detection risk is not adjusted and equals the audit risk. For a conservative analysis neglecting available prior information, the auditor can therefore ignore the ARM in its totality.

Despite the broad use of the ARM, it should be noted that it has been subject to criticism. First, detection risk can only be reduced, not eliminated, because of the stochastic nature of an audit. Second, the constituents of the audit risk are argued to influence each other, and should therefore not be treated as independent probabilities (Cushing and Loebbecke 1983). Multiplying these risks under an assumption of independence yields a 
model that is an inaccurate description of reality, and could expose the auditor to a higher level of audit risk than they would consider appropriate (Jiambalvo and Waller 1984; Kinney 1983). Instead, it is argued that the probabilities in the ARM are conditional (Leslie 1984), and that a model that considers these probabilities conditional on the evaluation of the preceding risks is a more accurate description of reality (Zaiceanu, Hlaciuc, and Lucan 2015). In such a model the audit is viewed as a continuous process in which the auditor formulates their beliefs on the prior probability of misstatement, conditional on their assessment of inherent risk and control risk (Leslie 1984). The auditor revises these beliefs as they collect audit evidence.

The aforementioned criticisms suggest that the ARM, as it is being used in practice, should be seen more as conceptual framework to incorporate prior information than as a mathematically correct model. Nonetheless, because the ARM is applied by a large number of accountancy firms in numerous countries, is well-known in the audit community, and is frequently mentioned throughout the ISA (IFAC 2010a,b,c) we have implemented the ARM in JfA to guide the statistical analyses.

\section{The Bayesian Approach to Auditing}

The Bayesian approach to auditing incorporates the prior knowledge and uncertainty about the probability of misstatement $\theta$ by a probability distribution $p(\theta)$ over its possible values. This is called the prior distribution. Using the available information in the sample (i.e., the likelihood $l($ data $\mid \theta))$, the prior distribution is transformed into a posterior probability distribution $p(\theta \mid$ data $)$ by means of Bayes' theorem. Consequently, the resulting estimate of the maximum misstatement is based on the combination of the information in the prior distribution and the information in the sample, see Equation 2.

$$
\underbrace{p(\theta \mid \text { data })}_{\text {Posterior }} \propto \underbrace{l(\text { data } \mid \theta)}_{\text {Likelihood }} \times \underbrace{p(\theta)}_{\text {Prior }}
$$


The auditor is not only interested in the most likely value of the misstatement, but also in the uncertainty associated with this estimate. The posterior distribution quantifies the uncertainty around the most likely value in the form of a credible bound. The $\left(1-\alpha_{0}\right)$ percent credible bound on the maximum misstatement is the $\left(1-\alpha_{0}\right)$ th percentile of the posterior distribution. The Bayesian credible bound can be interpreted as having a $\left(1-\alpha_{0}\right)$ percent probability of containing the true value $\theta$, since the values below it account for $(1-$ $\alpha_{0}$ ) percent of the posterior probability (Morey, Hoekstra, Rouder, Lee, and Wagenmakers 2016).

We consider the case of BuildIt again. Laura wants to know, with 95 percent confidence, whether BuildIt's financial statements contain misstatements lower than materiality. Laura has assessed the organization's inherent risk as high (100 percent), since BuildIt does not have an internal audit department. However, she found that the organization has a well-managed computer database and therefore assessed the control risk to be medium (60 percent). Consequently, imputing this information about the control risk in the ARM means that Laura can increase her required detection risk from $\alpha_{0}=5 \%$ to $\alpha_{1}=8.33 \%$. To continue her Bayesian audit, she specifies a prior distribution corresponding to the shift in detection risk. A detection risk of 5 percent corresponds to a sample size of 220 , and a detection risk of 8.33 percent corresponds to a sample size of 169 . Hence, incorporating the information on the control risk into the prior resulted in a reduction in sample size of 51 (see Appendix A for details).

After calculating the required sample size Laura uses MUS to select 169 monetary units from the population, and audits their corresponding transactions. Because she wants to incorporate the magnitude of the misstatement she chooses to calculate the taints of the transactions, see Equation 3. The taint $t_{i}$ of a transaction is the proportional difference between its book value $y_{i}$ and audit value $x_{i}$ : 


$$
t_{i}=\frac{y_{i}-x_{i}}{y_{i}}
$$

Positive taints are associated with overstatements, while negative taints occur when transactions are understated. In her selection Laura counts three overstatements, each having taint $t_{i}=0.6$. The next step is inferring an upper confidence bound on the population misstatement by evaluating the posterior distribution. Laura calculates the 95 percent credible estimate of the maximum misstatement in the population to be 2.77 percent. Consequently, her estimate of the maximum estimated population misstatement is $\$ 38,802$, which is lower than the materiality of $\$ 70,160$, allowing her to approve BuildIt's financial statements.

In auditing, as in many fields of science, the major difference between the currently dominating frequentist methods and their Bayesian alternatives is that in a Bayesian analysis, expert- or prior knowledge can be incorporated in the statistical analysis in a principled manner by constructing a prior distribution reflecting this knowledge (Beck, Solomon, and Tomassini 1985; Corless 1972; Felix 1976; Crosby 1981; Dyer and Pierce 1993; Gronau, Ly, and Wagenmakers 2019; Meeden and Sargent 2007; Van Batenburg, O’Hagan, and Veenstra 1994). When the auditor has constructed this prior distribution, it allows her to make a population statement that is more fitting in an audit context than a frequentist one. The reason why this statement is more fitting is that the Bayesian statement is based on the specific audit, while the frequentist statement is based on a theoretical long-term frequency over repeated audits (Bayarri and Berger 2004; Pratt 1965; Wagenmakers, Lee, Lodewyckx, and Iverson 2008). Other than the difference in interpretation, Bayesian methods are more flexible in the sense that they allow an auditor to statistically update her statement about the probability of material misstatement in the population in the face of new information, something that is not allowed in the frequentist framework (Etz, Gronau, Dablander, Edelsbrunner, and Baribault 2018; Kass and Raftery 1995; O’Hagan 2004; Rouder 2014). To completely explain the 
differences between the frequentist and Bayesian approaches is beyond the scope of this paper, and we refer the reader to the cited literature for more information.

\section{JFA: JASP FOR AUDIT}

JASP for Audit (JfA) is an add-on module for JASP, a free and open-source statistical software package with a point-and-click graphical user interface that runs on the statistical programming language $\mathrm{R}$ ( $\mathrm{R}$ Core Team 2019). The goal of JfA is to offer the auditor guidance in planning, performing, and interpreting their audit. This is done by implementing the most commonly used statistical auditing techniques in a standardized audit workflow that relates directly to familiar audit concepts and the ISA. In addition, JfA offers a frequentist and a Bayesian version of this workflow. The Bayesian techniques enable the auditor to take advantage of the benefits of knowledge updating, which were previously not readily available to use in an audit context.

In this section we will discuss how the structure of JfA facilitates the auditor in their workflow. To illustrate the functionality of JfA, we will discuss a monetary walkthrough of a Bayesian audit.

\section{The Audit Workflow in JASP}

The audit workflow in JASP can be found by clicking the "Audit" module icon and selecting "Audit Workflow" for the frequentist workflow or "Bayesian Audit Workflow" for its Bayesian counterpart. Both workflows are aligned with the audit workflow as discussed in sections two and three as much as possible. Consequently, starting one of these workflows will present the auditor with the planning stage, displayed in Figure 1. 
In these interactive layouts the auditor can select the required data and options to perform their audit. All stages have output in the form of tables and plots that clarify the statistical results, which can be found under the "Tables and Plots" section. Upon completion of a stage, the auditor can request a report of the output by clicking the "Download Report" button (greyed out in Figure 1). When the auditor has finished the planning stage they can move on to the selection stage by clicking the "To Selection" button (greyed out in Figure 1), which becomes available once all required input has been specified. These buttons can be found at the bottom of every stage and progress the auditor through the workflow. However, the auditor is free to move between stages that are completed until the execution stage is reached, as they may wish to make changes to their audit design. The selection, execution, and evaluation stages have a similar layout as the planning stage to keep the auditor focused on their matter of expertise.

Figure 2 summarizes JfA's bare-bones audit workflow, showing each stage as a stacked block with inputs and outputs. The input blocks show the specific data and options the auditor has to specify to perform their audit. The output blocks show the specific tables, statistics, and plots that the auditor is able to request from JfA in the corresponding stages.

The input and output for future audit stages are conditional on the statistical choices made in previous stages. This conditionality of the audit process enables JfA to guide the auditor in their workflow. For example, if the auditor drags a column of book values to the corresponding data input in the planning stage, JfA will automatically switch to MUS in the selection stage. A complete flowchart of JfA's branching is displayed in Figure 3.

In the next sections we consider the case of BuildIt again. Using a monetary example, we will show how to perform an audit of BuildIt's organization using JfA. We will 
familiarize the reader with the different input and output options in JfA by highlighting the important ones in snapshots of the program.

\section{Example to Detect Overstatements}

In this example we consider the case where the auditor is auditing financial statements. The data for this example represents BuildIt's financial statements and consists of 3500 observations of record ID's, book values, and their corresponding audit values. The audit values are added for illustrative purposes, since the auditor would have to assess this information in the execution stage of the audit. A reproducible .jasp file containing the data and carried-out analysis (BuildIt Monetary.jasp) can be found in JASP's internal data library. For this example audit, a population materiality of $\$ 70,161$ (five percent) applies.

\section{Stage 1: Planning}

The auditor starts the Bayesian audit workflow in the planning stage. Figure 4 is an annotated snapshot of the input screen for the planning stage, which we will use to explain how audit concepts are presented to the auditor in JfA. Reading from left to right and top to bottom, the auditor is first instructed to state the population materiality as an absolute monetary amount or a percentage relative to the total value in box one. The desired confidence level can be set in box two. For this example audit a default confidence level of 95 percent is attained, setting the detection risk to five percent. The auditor can now insert their record ID's and book values (which are required when the materiality is specified as an absolute amount) in box three. For a monetary audit, the auditor can also specify their preferred currency. Prior to the substantive testing stage, the risk assessments for inherent risk and control risk were determined to be high (100 percent) and medium (60 percent), which the auditor can specify with the buttons in box four. As a result of this information, the detection risk is increased to 8.33 percent. Based on last year's audit, at most 2.5 percent 
errors are expected in the population and the auditor can enter this relative number in box five. The distribution that is used for planning the audit can be specified in box six and defaults to the beta distribution from section three. Explanatory text that helps the auditor in interpreting the results can be displayed throughout the analysis by enabling box seven. When all required fields are specified, JfA will calculate the required sample size and present it to the auditor as in Figure 5.

From the "Tables and Plots" section the auditor can request output that describes the prior distribution. The bottom table in Figure 5 shows the implicit sample on which the prior distribution is based, which for the current example consists of 51 observations of which 1.28 were assumed to contain an error. The plot visualizes the prior distribution that corresponds with this implicit sample, and the expected posterior distribution, which has its 95th percentile just below the materiality. With the required sample size from Figure 5 in mind the auditor can continue to the selection stage by clicking the button "To Selection".

\section{Stage 2: Selection}

Figure 6 is an annotated snapshot of the input screen for the selection stage. Upon entering the selection stage, JfA automatically selects the required subset from the population using the default fixed interval sampling method. Since the auditor has provided book values in the planning stage, JfA automatically enables and selects MUS by default. Under "Advanced Options" the auditor has the choice of selecting different sampling units in box one, and a different sampling method in box two. To make results reproducible, a random number generator seed is by default specified as one, but can be changed in box three. For comparison with section two and three, the seed in this example is set to one. The results of the selection stage are presented to the auditor as in Figure 7.

\section{Stage 3: Execution}


In the execution stage, the auditor must define how they will annotate their selection. Figure 8 is an annotated snapshot of the input screen for the execution stage. The auditor can specify their preferred annotation method in box one. Since audit values are the preferred annotation methods for book values, JfA selects this option by default.

Two columns will be added to the data set during this stage. The first column will contain the results of the selection stage, indicating whether a transaction is included or excluded from the selection and how many times it should be counted as a result of selecting with replacement. The second column will be empty, but must be filled by the auditor with the results of their audit. Upon providing names for the columns in box two, and pressing the "Fill Variables" button in box three, these columns will be pasted to the data. After filling the audit result column for the selected transactions in the data editor that opens up in box four, the auditor can proceed to the evaluation stage by clicking the "To Evaluation" button.

\section{Stage 4: Evaluation}

Figure 9 is an annotated snapshot of the input screen for the Bayesian evaluation stage. The selection result column is recognized automatically by JfA and transactions are counted automatically without any effort. For this example, JfA automatically displays and chooses the appropriate statistical evaluation method for monetary annotations in box two. When the auditor provides their audit result column in box one, the statistical results are automatically computed, and a population statement is immediately printed as a conclusion. The results from the evaluation stage are presented to the auditor as in Figure 10, confirming the estimate of the estimated maximum misstatement in sections two and three. To clarify these results the auditor can request several statistics, tables, and plots from the "Tables and Plots" section. 
A plot of the prior and posterior distribution can be informative for the auditor, as it visualizes the uncertainty in their estimate of the misstatement. The posterior distribution can also be used to construct a $\left(1-\alpha_{0}\right)$ percent credible interval, which can be selected in box three. The credible interval is the area around the mode of the posterior that contains $(1-$ $\alpha_{0}$ ) percent of the probability, and can facilitate discussion about the continuation of the audit by quantifying two-sided uncertainty about the population misstatement.

To clarify their results in relation to the materiality, the auditor may choose to display an evaluation information plot. This plot visualizes the materiality, maximum error, and most likely error (MLE) as a horizontal bar chart. The graph should make it easy for auditors to determine whether the estimated maximum misstatement is lower than the materiality, as the color of its bar switches between red and green depending on whether the estimate is respectively above or below the materiality. For this example, the 95 percent credible bound on the misstatement is below the materiality. Consequently, the conclusion of this audit is that the data support the assertion that the population contains less than five percent misstatements.

\section{CONCLUDING COMMENTS}

In this article we introduced JASP for Audit (JfA), an open-source software package designed specifically for statistical auditing. With JfA, we set out to increase audit quality and decrease audit complexity. The module decreases audit complexity because audit analyses are readily available in a standardized workflow that should feel intuitive to auditors. The interface is user-friendly, and directly relates to audit processes and to the International Standards of Auditing (IFAC 2010a,b,c,d,e). Furthermore, JfA may increase efficiency and knowledge of audit theory by taking over the statistical heavy lifting from the auditor and 
guiding them through the audit process, thereby having the potential to increase audit quality by preventing common mistakes in planning, selecting, executing, and interpreting the sample (Brivot et al. 2018).

With the heightened focus on audit quality by both regulators and society, auditors must be able to provide their statements with appropriate justification and comfort, while they experience the pressure of time and audit fees. A reliance on complicated, non-adjustable, software reduces the time auditors can spend on other audit activities. JfA streamlines the auditor's sampling procedure so that they can focus on their matter of expertise. Furthermore, the addition of Bayesian inference in JfA allows the auditor to effectively incorporate many sources of prior information into their audit design, ensuring maximum use of the available knowledge. Especially in light of current audit failures (IFIAR 2018; van Brenk et al. 2019) such knowledge may be used to create an informed sampling procedure.

JfA's free open-source structure leads to two benefits. First, it reduces audit costs, as it eliminates the need for audit companies to buy software licenses. Second, since it is opensource, audit firms can add audit firm specific methods to JfA, or tailor the documentation to their specific needs. For example, an audit firm may want to make references to their audit guide in the output annotation. Last but not least, JfA automatically generates the audit conclusions in both text, tables, and graphical formats, including an audit trail in the language of the auditor.

In sum, JfA can be a valuable addition to the audit software curriculum. As we expect the number of audits to grow in the future, our hope is that JfA will help increase their quality and reduce the time and money spent per audit. 


\section{REFERENCES}

AICPA (2017). Audit Guide: Audit Sampling. American Institute of Certified Public Accountants.

Alles M. G., A. Kogan, and M. A. Vasarhelyi. 2008. Putting continuous auditing theory into practice: Lessons from two pilot implementations. Journal of Information Systems 22 (2): $195-214$.

AlMarzouq M., L. Zheng, G. Rong, and V. Grover. 2005. Open source: Concepts, benefits, and challenges. Communications of the Association for Information Systems 16 (1): 756-784.

Appelbaum D., A. Kogan, and M. A. Vasarhelyi, 2017. Big Data and analytics in the modern audit engagement: Research needs. Auditing: A Journal of Practice \& Theory 36 (4): 1-27.

Bayarri M. J., and J. O. Berger. 2004. The interplay of Bayesian and frequentist analysis. Statistical Science 19 (1): 58-80.

Beasley M. S., J. V. Carcello, and D. R. Hermanson. 2001. Top 10 audit deficiencies. Journal of Accountancy 19 (1): 63-66.

Beck P. J., I. Solomon, and L. A. Tomassini. 1985. Subjective prior probability distributions and audit risk." Journal of Accounting Research 21 (1): 37-56.

Bedingfield J.P. 1975. The current state of statistical sampling and auditing. Journal of Accountancy 140 (6): 48.

Brivot, M., M. Roussy, and M. Mayer. 2018. Conventions of audit quality: The perspective of public and private company audit partners. Auditing: A Journal of Practice \& Theory 37 (2): 51-71. 
Brown-Liburd, H., H. Issa, and D. Lombardi. 2015. Behavioral implications of Big Data's impact on audit judgment and decision making and future research directions. Accounting Horizons 29 (2): 451-468.

Burgstahler D., and J. Jiambalvo. 1986. Sample error characteristics and projection of error to audit populations. Accounting Review 61 (2): 233-248.

Cao M, R. Chychyla, and T. Stewart. 2015. Big data analytics in financial statement audits. Accounting Horizons 29 (2): 423-429.

Caseware Analytics. 2018. IDEA [Computer Software].

Christensen B. E., R. J. Elder, and S. M. Glover. 2015. Insights into large audit firm sampling policies. Current Issues in Auditing 9 (2): 7-18.

Corless J. C. 1972. Assessing prior distributions for applying Bayesian statistics in auditing. The Accounting Review 47 (3): 556-566.

Crosby M. A. 1981. Bayesian statistics in auditing: A comparison of probability elicitation techniques. Accounting Review 56 (2): 355-365.

Cushing B. E., and J. K. Loebbecke. 1983. Analytical approaches to audit risk: A survey and analysis. Auditing: A Journal of Practice \& Theory 3 (1): 23-41.

De George E. T., C. B. Ferguson, and N. A. Spear. 2012. How much does IFRS cost? IFRS adoption and audit fees. The Accounting Review 88 (2): 429-462.

De Swart J., J. Wille, and B. Majoor. 2013. Het 'push left'-principe als motor van data analytics in de accountantscontrole [The 'push-left'-principle as a driver of data analytics in financial audit]. Maandblad voor Accountancy en Bedrijfseconomie 87: 425-432. 
Durney M., R. J. Elder, and S. M. Glover. 2014. Field data on accounting error rates and audit sampling. Auditing: A Journal of Practice \& Theory 33 (2): 79-110.

Dyer D., and R. L. Pierce. 1993. On the choice of the prior distribution in hypergeometric sampling. Communications in Statistics - Theory and Methods 22 (8): 2125-2146.

Dzuranin A. C., J. R. Jones, and R. M. Olvera. 2018. Infusing data analytics into the accounting curriculum: A framework and insights from faculty. Journal of Accounting Education 43: $24-39$.

Earley C. E. 2015. Data analytics in auditing: opportunities and challenges. Business Horizons 58 (5): 493-500.

Elder R. J., A. D. Akresh, S. M. Glover, J. L. Higgs, and J. Liljegren. 2013. Audit sampling research: A synthesis and implications for future research. Auditing: A Journal of Practice \& Theory 32 (1): 99-129.

Etz A., Q. F. Gronau, F. Dablander, P. A. Edelsbrunner, and B. Baribault. 2018. How to become a Bayesian in eight easy steps: An annotated reading list. Psychonomic Bulletin \& Review 25 (1): 219-234.

Felix W. L. 1976. Evidence on alternative means of assessing prior probability distributions for audit decision making. The Accounting Review 51 (4): 800-807.

Felix W. L., and W. R. Kinney 1982. Research in the auditor's opinion formulation process: State of the art. Accounting Review 57 (2): 245-271.

Fienberg S. E., J. Neter, and R. A. Leitch. 1977. Estimating the total overstatement error in accounting populations. Journal of the American Statistical Association 72 (358): 295-302.

Galvanize. 2018. ACL [Computer Software]. 
Glover, S. M., M. H. Taylor, and Y. Wu. 2017. Current practices and challenges in auditing fair value measurements and complex estimates: Implications for auditing standards and the academy. AUDITING: A Journal of Practice \& Theory 36 (1): 63-84.

Gronau Q. F., A. Ly, and E.-J. Wagenmakers. 2019. Informed Bayesian t-tests. The American Statistician.

Héroux M. 2016. Inadequate reporting of statistical results. Journal of Neurophysiology 116 (3): 1536-1537.

Hitzig N. B. 1995. Audit sampling: A survey of current practice. The CPA Journal 65 (7): 5457.

International Federation of Accountants (IFAC). 2010a. International standard on auditing 200: Overall objectives of the independent auditor and the conduct of an audit in accordance with international on auditing. Available at: https://www.ifac.org/system/files/downloads/a008-2010-iaasb-handbook-isa-200.pdf International Federation of Accountants (IFAC). 2010b. International standard on auditing 315: Identifying and assessing the risks of material misstatement through understanding the entity and its environment. Available at: https:/www.ifac.org/system/ files/downloads/a017-2010-iaasb-handbook-isa-315.pdf.

International Federation of Accountants (IFAC). 2010c. International standard on auditing 320: Materiality in planning and performing an audit. Available at: https://www.ifac.org/system/files/downloads/a018-2010-iaasb-handbook-isa-320.pdf.

International Federation of Accountants (IFAC). 2010d. International standard on auditing 450: Evaluation of misstatements identified during the audit." Available at: https://www.ifac.org/system/files/downloads/a021-2010-iaasb-handbook-isa-450.pdf. 
International Federation of Accountants (IFAC). 2010e. International standard on auditing 530: Audit sampling. Available at: https://www.ifac.org/system/files/downloads/a027-2010iaasb-handbook-isa-530.pdf

International Forum of Independent Audit Regulators (IFIAR). 2018. Survey of inspection findings 2017. Retrieved from https://www.ifiar.org/?wpdmdl=7970

Interdepartementaal Overlegorgaan Departementale Accountantsdiensten (IODAD). 2007. Handboek auditing rijksoverheid 2007.

JASP Team (2018). JASP (Version 0.10.0)[Computer software].

Jiambalvo J., and W. Waller. 1984. Decomposition and assessments of audit risk. Auditing: A Journal of Practice \& Theory 3 (2): 80-88.

Johnstone D. 1997. Comparative classical and Bayesian interpretations of statistical compliance tests in auditing. Accounting and Business Research 28 (1): 53-82.

Kass R. E., and A. E. Raftery. 1995. Bayes factors. Journal of the American Statistical Association 90 (430): 773-795.

Kinney W. R. 1983. A note on compounding probabilities in auditing. Auditing: A Journal of Practice \& Theory 2 (2): 13-22.

Knechel, R. W. 2007. The business risk audit: Origins, obstacles and opportunities. Accounting, Organizations and Society 32 (4-5): 383-408.

Kogan, A., B. W. Mayhew, and M. A. Vasarhelyi. 2019. Audit data analytics research-An application of design science methodology. Accounting Horizons 33 (3): 69-73. 
Leslie D. A. 1984. An analysis of the audit framework focusing on inherent risk and the role of statistical sampling in compliance testing. Research on Auditing Symposium VII. University of Kansas.

Li H., J. Dai, T. Gershberg, and M. A. Vasarhelyi. 2018. Understanding usage and value of audit analytics for internal auditors: An organizational approach. International Journal of Accounting Information Systems 28: 59-76.

Love J., R. Selker, M. Marsman, T. Jamil, D. Dropmann, J. Verhagen, A. Ly, Q. F. Gronau, M. Smira, S. Epskamp, D. Matzke, A. Wild, P. Knight, J. N. Rouder, R. D. Morey, and E.J. Wagenmakers. 2018. JASP: Graphical statistical software for common statistical designs. Journal of Statistical Software 88 (2).

Maxwell E. 2006. Open standards, open source, and open innovation: Harnessing the benefits of openness. Innovations: Technology, Governance, Globalization 1 (3): 119-176.

McAfee A., E. Brynjolfsson, T. H. Davenport, D. Patil, and D. Barton. 2012. Big data: The management revolution. Harvard Business Review 90 (10): 60-68.

Meeden G., and D. Sargent. 2007. Some Bayesian methods for two auditing problems. Communications in Statistics - Theory and Methods 36 (15): 2727-2740.

Morey R. D., R. Hoekstra, J. N. Rouder, M. D. Lee, and E.-J. Wagenmakers. 2016. The fallacy of placing confidence in confidence intervals. Psychonomic Bulletin \& Review 23 (1): 103123.

Neter J., and K. James. 1975. Behavior of major statistical estimators in sampling accounting populations: An empirical study; Auditing research monograph, 2. Guides, Handbooks and Manuals 9. 
Niemi L. 2005. Audit effort and fees under concentrated client ownership: Evidence from four international audit firms. The International Journal of Accounting 40 (4): 303-323.

Nieschwietz R., K. Pany, and J. Zhang. 2002. Auditing with technology: Using generalized audit software in the classroom. Journal of Accounting Education 20 (4): 307-329.

O’Hagan, A. 2004. Bayesian statistics: Principles and benefits. Frontis 3: 31-45.

Pratt J. W. 1965. Bayesian interpretation of standard inference statements. Journal of the Royal Statistical Society: Series B (Methodological) 27 (2): 169-203.

Ramachandran Rackliffe U., and L. Ragland. 2016. Excel in the accounting curriculum: Perceptions from accounting professors. Accounting Education 25 (2): 139-166.

Ramage J. G., A. M. Krieger, and L. L Spero. 1979. An empirical study of error characteristics in audit populations. Journal of Accounting Research 17: 72-102.

R Core Team. 2019. R: A language and environment for statistical computing. R Foundation for Statistical Computing, Vienna, Austria.

Rouder J. N. 2014. Optional stopping: No problem for Bayesians. Psychonomic Bulletin \& Review 21 (2): 301-308.

Ruhnke K., and M. Schmidt. 2014. Misstatements in financial statements: The relationship between inherent and control risk factors and audit adjustments. Auditing: A Journal of Practice \& Theory 33 (4): 247-269.

Schwartz D. A. 1997. Audit sampling - A practical approach. The CPA Journal 67 (2): 56-59.

Sledgianowski D., M. Gomaa, and C. Tan. 2017. Toward integration of big data, technology and information systems competencies into the accounting curriculum. Journal of Accounting Education 38: 81-93. 
Stewart T. 2013. A Bayesian audit assurance model with application to the component

materiality problem in group audits.

Swanepoel, E. 2018. The effectiveness of audit sampling methods recommended to small and medium practices in guides published by audit regulators. South African Journal of Accounting Research 32 (2-3): 109-131.

TheWorldBank. 2019. Listed domestic companies. Available at: https://data.worldbank.org/indicator/CM.MKT.LDOM.NO

Titera W. R. 2013. Updating audit standard - enabling audit data analysis. Journal of Information Systems 27 (1): 325-331.

Van Batenburg P. C., A. O’Hagan, and R. H. Veenstra. 1994. Bayesian discovery sampling in financial auditing: A hierarchical prior model for substantive test sample sizes. Journal of the Royal Statistical Society: Series D (The Statistician) 43 (1): 99-110.

Van Brenk, H., R. Renes, and G. Trompeter. 2019. Auditing in the public interest: Reforming the profession by building on the strengths of the existing accounting firms. SSRN Electronic Journal.

Wagenmakers E.-J., M. D. Lee, T. Lodewyckx, and G. J. Iverson. 2008. Bayesian versus frequentist inference. In Bayesian evaluation of informative hypotheses: 181-207. Springer

Wasserstein R. L., and N. A. Lazar. 2016. The ASA's statement on p-values: Context, process, and purpose. The American Statistician 70 (2): 129-133.

Webb T. Z., and T. R. Waymire. 2016. Large sample evidence of the determinants of nonprofit monitoring costs: A resource dependence framework. Journal of Governmental \& Nonprofit Accounting 5 (1): 25-52. 
Weidenmier, M. L., and T. L. Herron. Selecting an audit software package for classroom use. Journal of Information Systems 18 (1): 95-110.

Wolters Kluwers. 2018. TeamMate Analytics [Computer Software]. 


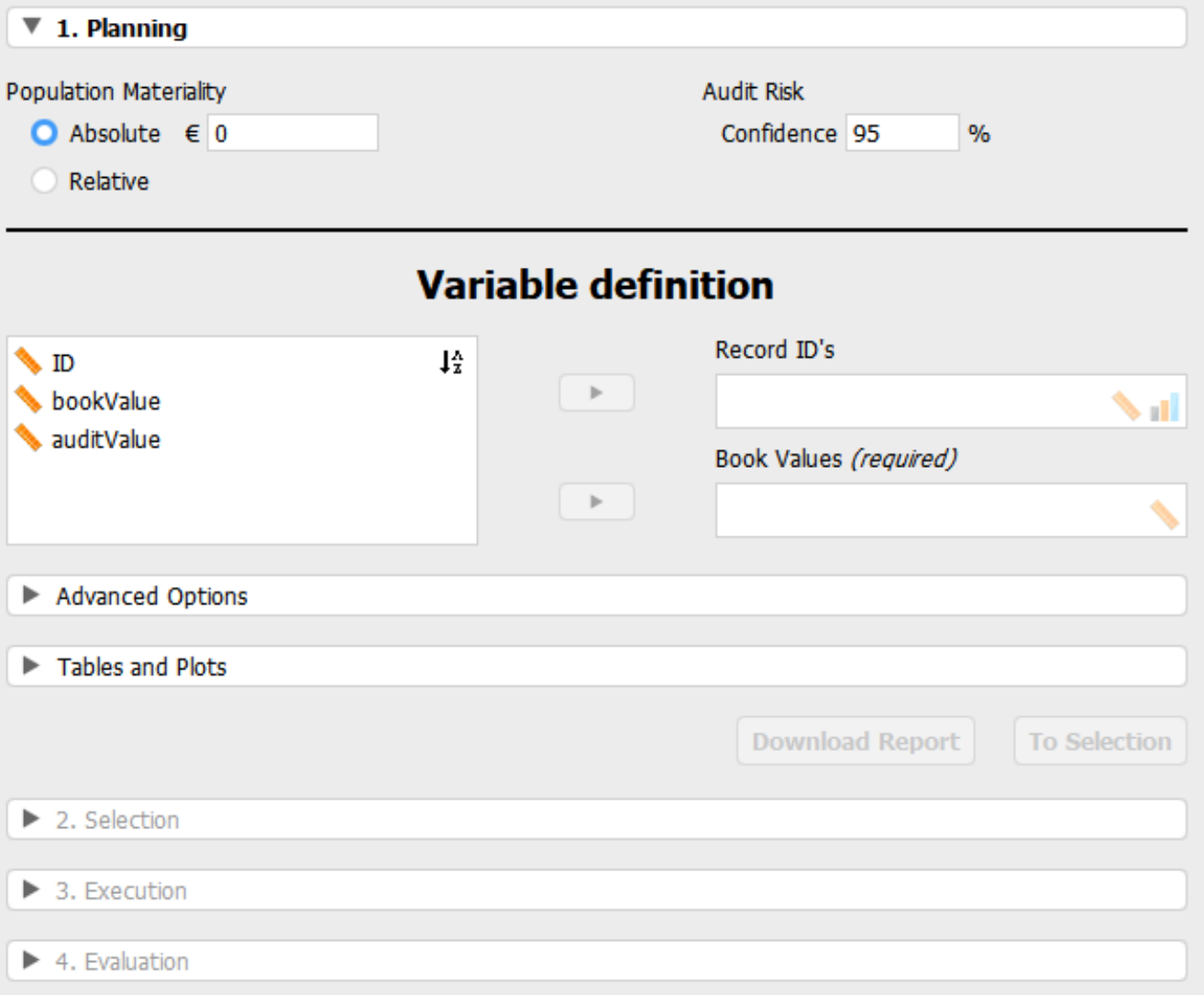

Figure 1: Snapshot of the input screen for the planning stage of JfA's audit workflow. The future selection, execution and evaluation stages are presented as (greyed out) bars that expand when the auditor is ready to move on. The interface directly relates to audit concepts, like the population materiality, so that it is easy for auditors to recognize and locate relevant options. 


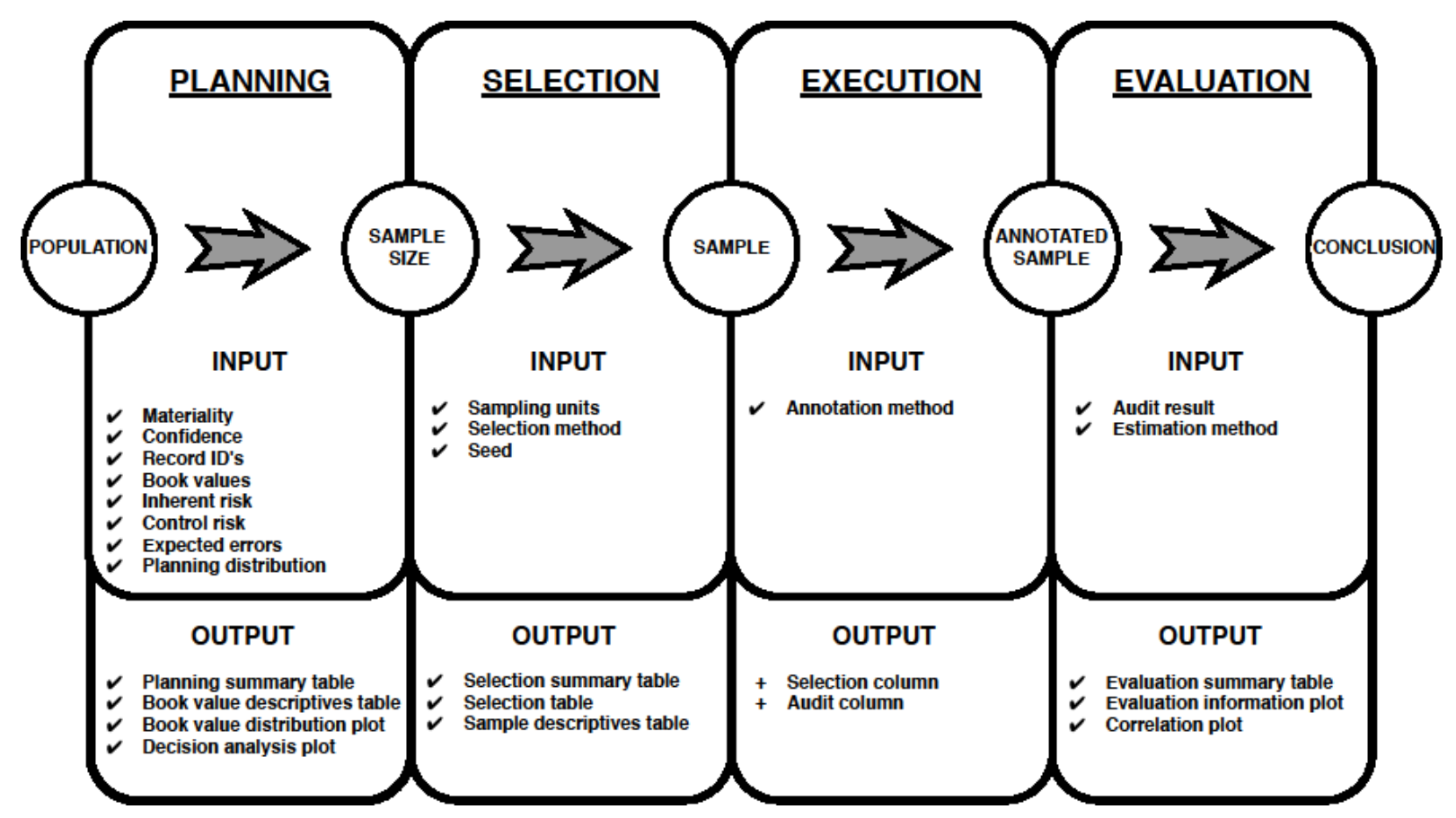

Figure 2: JfA's audit workflow displayed as progress chart, showing each stage as a block with inputs and outputs. The circles represent the flow of data, emphasizing that the output of previous stages is used as input for the future ones. Most input options have sensible default values in accordance with the ISA, requiring the auditor to make adjustments only when needed. 


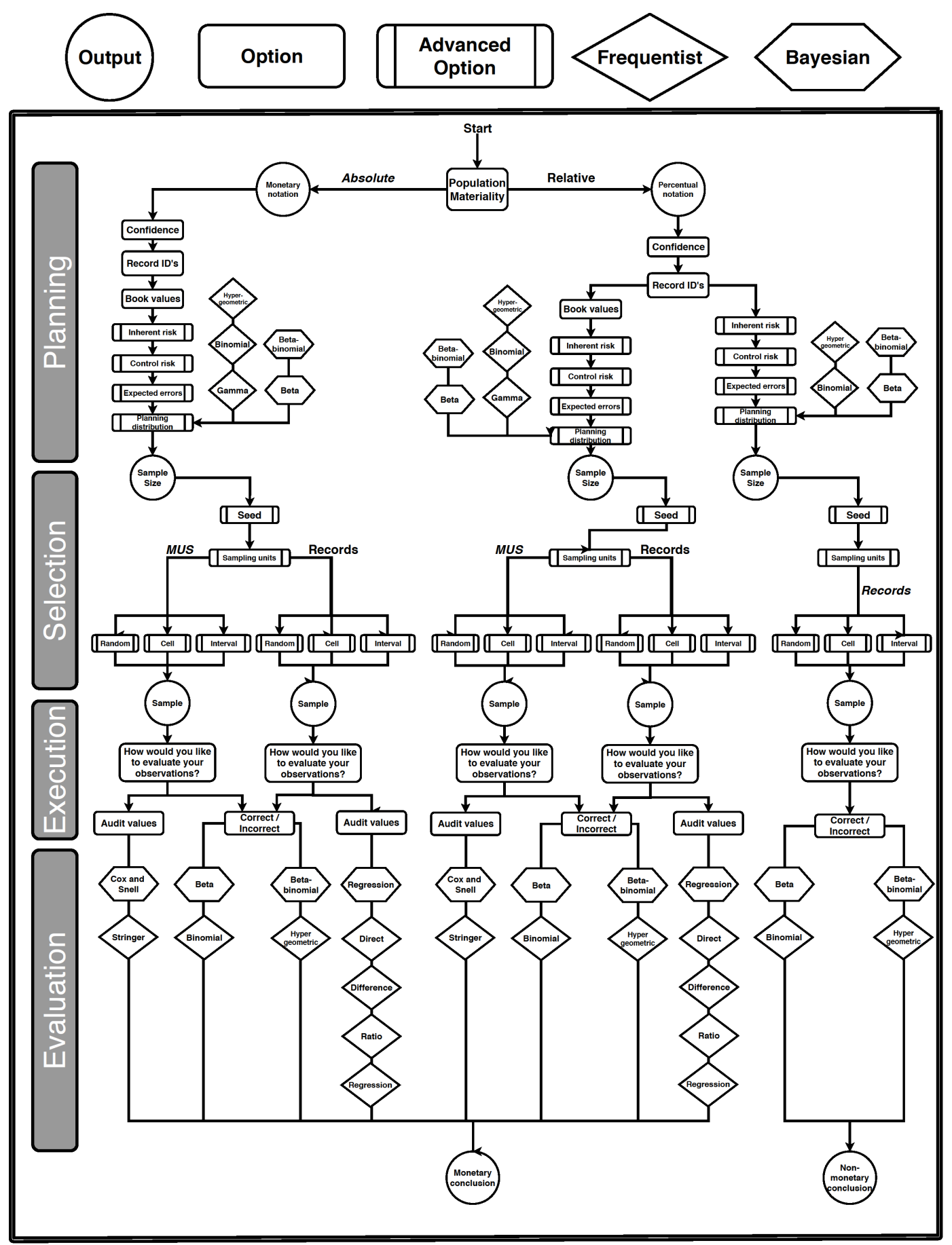

Figure 3: JfA's audit workflow displayed as a flowchart, showing the possible paths that an auditor takes conditional on the options chosen in the previous stages. The circles represent the most relevant output that JfA returns. Other boxes represent input options, making a distinction between regular options (rectangles), advanced options (extended rectangles) and options that are only available in the frequentist (diamonds) and Bayesian (hexagons) workflows. Default options are displayed in italic. 


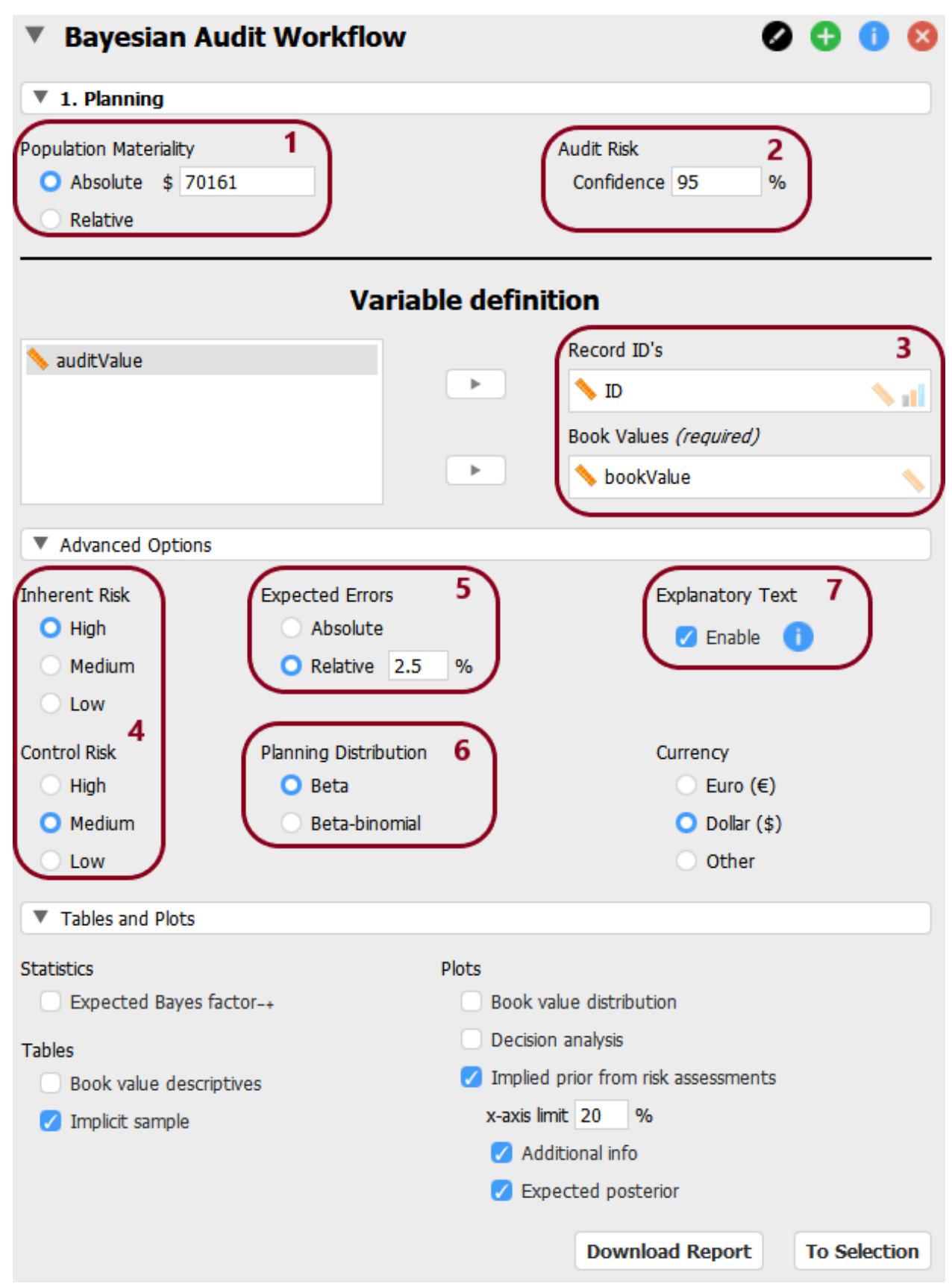

Figure 4: Snapshot of the input screen for JfA's Bayesian planning stage. Box one represents the population materiality and box two represents the confidence level for the analysis. The auditor can insert their record ID's in box three. The risk assessments from the ARM may be given in box four and the auditor can specify their expected errors in box five. The assumed statistical distribution used for the calculations can be specified in box six. Finally, the auditor can enable explanatory text throughout every stage of the workflow by enabling the checkbox in box seven. 


\section{Planning}

The most likely error in the data was expected to be $2.5 \%$. The sample size that is required to prove a materiality of $\$ 70161$, assuming the sample contains $2.5 \%$ full errors, is 169 . This sample size is calculated according to the beta distribution, the inherent risk (High),

the control risk (Medium) and the expected errors. The specific distribution that corresponds with this prior knowledge is the

Beta(2.28,50.73) distribution. The information in this prior distribution states that there is a $67.25 \%$ prior probability that the population misstatement

is lower than materiality. Consequently, if the sum of errors from the audited observations exceeds 5 the maximum misstatement

exceeds materiality and the population cannot be approved

Planning Summary

\begin{tabular}{llllll}
\hline Materiality & Inherent risk & Control risk & Detection risk & Expected errors & Required sample size \\
\hline S 70161 & High & Medium & $8.3 \%$ & 4.23 & 169 \\
\hline Note. The required sample size is based on the beta distribution $(\alpha=2.28, \beta=50.73)$.
\end{tabular}

Implicit Sample

Implicit sample size Implicit errors $95 \%$ Prior credible bound

$51 \quad 9.28 \%$

Note. Sample sizes shown are implicit sample sizes derived from the

ARM risk assessments: $\mathrm{IR}=$ High and $\mathrm{CR}=$ Medium

Implied Prior from Risk Assessments

$$
\text { - - Prior … Expected posterior }
$$

$95 \%$ Prior

credible region

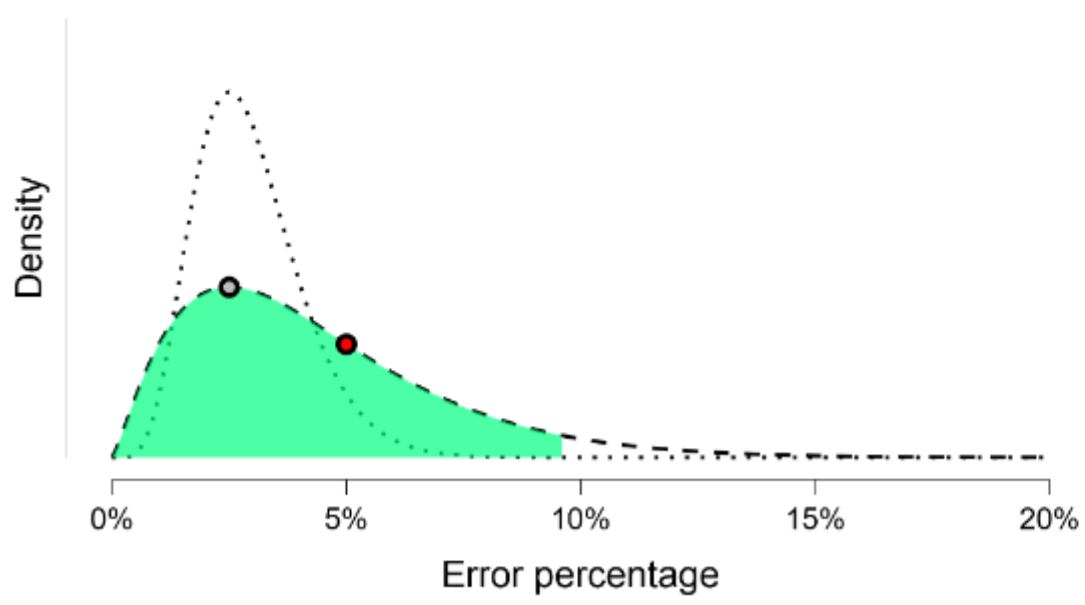

Figure 1. The prior probability distribution (beta) on the misstatement in the population. The expected errors (grey) receive the highest probability. The red dot represents the materiality. The prior parameters $\alpha=2.275, \beta=50.725$ are derived from the assessments of the inherent and control risk, along with the expected errors.

The expected posterior has its upper confidence bound below materiality.

Figure 5: Snapshot of the output screen of JfA's Bayesian planning stage containing the planning summary table and an annotation explaining the planning process. Also included are the prior distribution on the population misstatement, and the implicit sample table on which the prior is based. The red dot represents the specified materiality. The grey dot represents the expected errors. 


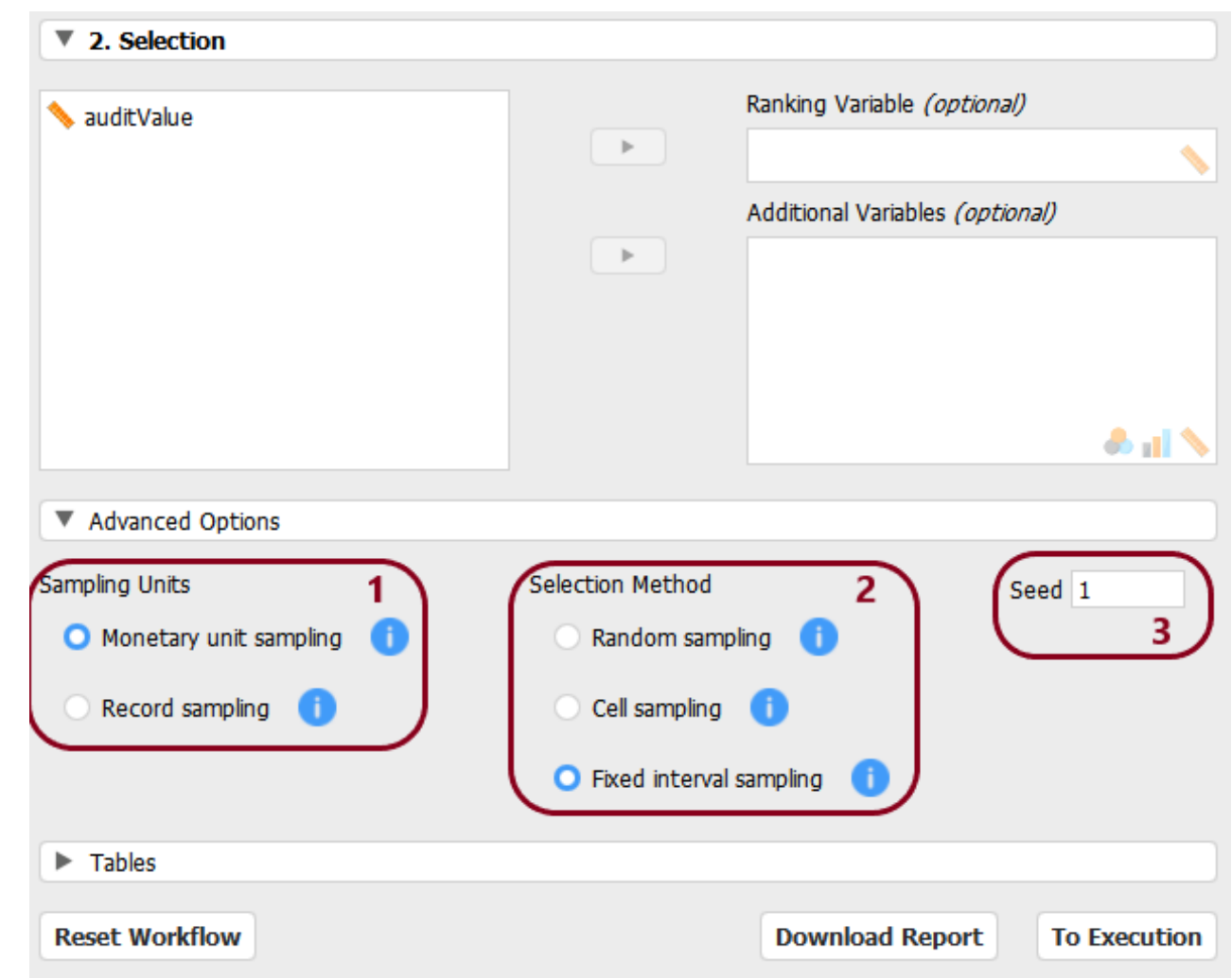

Figure 6: Snapshot of the input screen for JfA's selection stage. The radio buttons in box one enable the auditor to choose between MUS and record sampling. The sampling scheme can be specified in box two and a seed can be set in box three to reproduce results. 


\section{Selection}

From the population of 3500 observations, 169 observations were selected using a fixed interval monetary unit sampling method.

Selection Summary

\begin{tabular}{llll}
\hline Sample size & Sample value & $\%$ of total value & Interval \\
\hline 169 & $\$ 84424$ & $6.02 \%$ & $\$ 8304$ \\
\hline
\end{tabular}

Note. The sample is drawn with seed 1 .

Figure 7: Snapshot of the output screen of JfA's selection stage containing the selection summary table and an annotation explaining the selection process. 


\section{Execution}

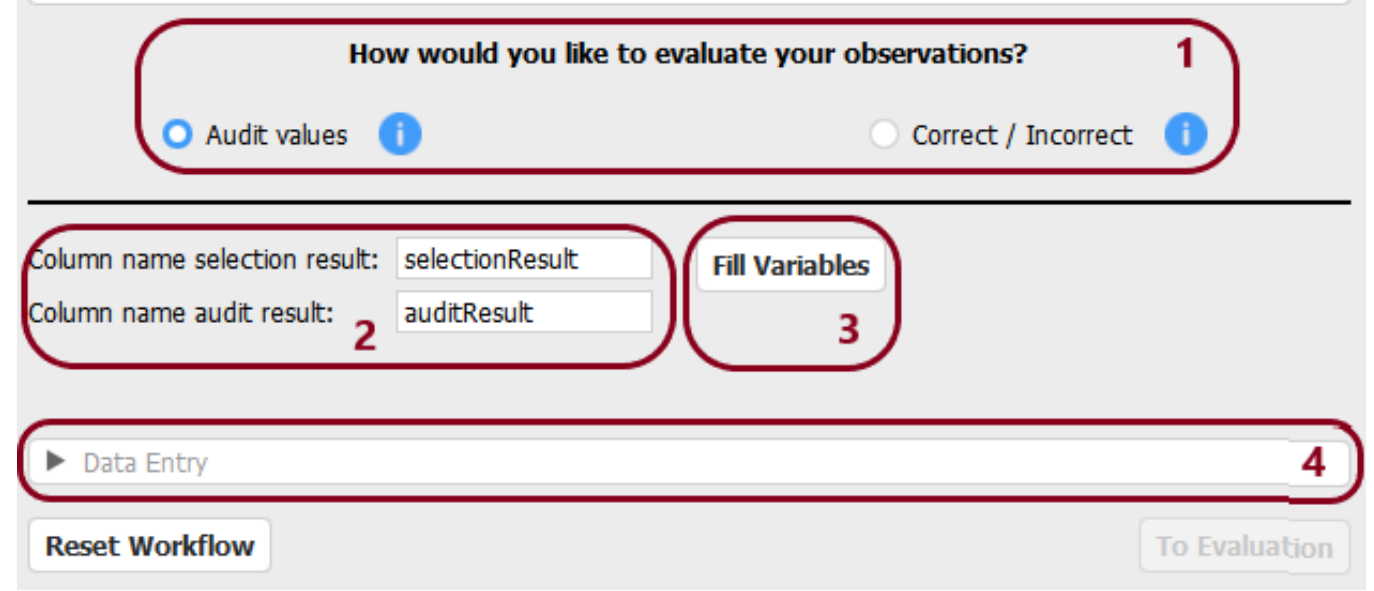

Figure 8: Snapshot of the input screen for JfA's execution stage. The auditor chooses how to annotate their observations in box one and provides names in box two for the columns that will be added. By clicking the button in box three, these columns are pasted to the data set and are editable in box four. 
4. Evaluation

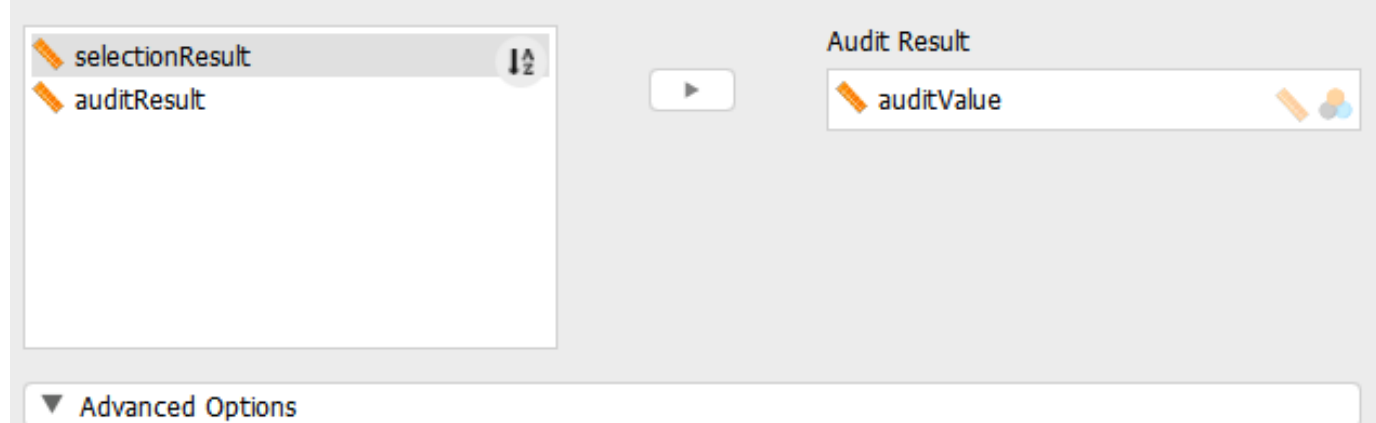

Advanced Options

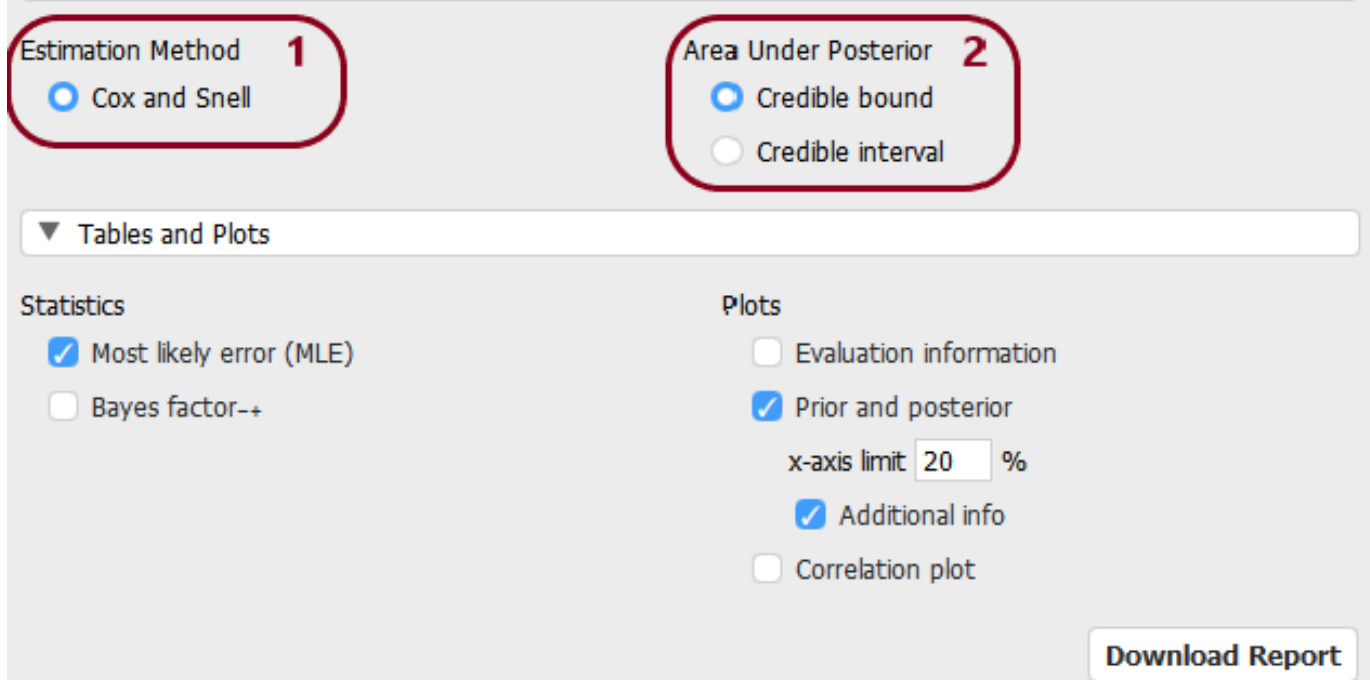

Figure 9: Snapshot of the input screen for JfA's Bayesian evaluation stage. The audit result must be inserted in box one. The estimation method can be chosen in box two. Several statistics and plots to clarify the evaluation result can be requested from the "Tables and Plots" section. 


\section{Evaluation}

The selection consisted of 169 observations, 3 of which were found to contain an error. The knowledge from these data, combined with the prior knowledge results in an $95 \%$ upper confidence bound of $2.77 \%$. The cumulative knowledge states that there is a true probability of $95 \%$ that the misstatement in the population is lower than $2.77 \%$.

Evaluation Summary

\begin{tabular}{lllllll}
\hline Materiality & Sample size & Errors & Total tainting & MLE & $95 \%$ Credible bound & Maximum Misstatement \\
\hline \$ 70161 & 169 & 3 & 1.8 & $0.62 \%$ & $2.77 \%$ & $\$ 38802$ \\
\hline Note. The credible bound is calculated according to the Cox and Snell method
\end{tabular}

Prior and Posterior

- - Prior - Posterior
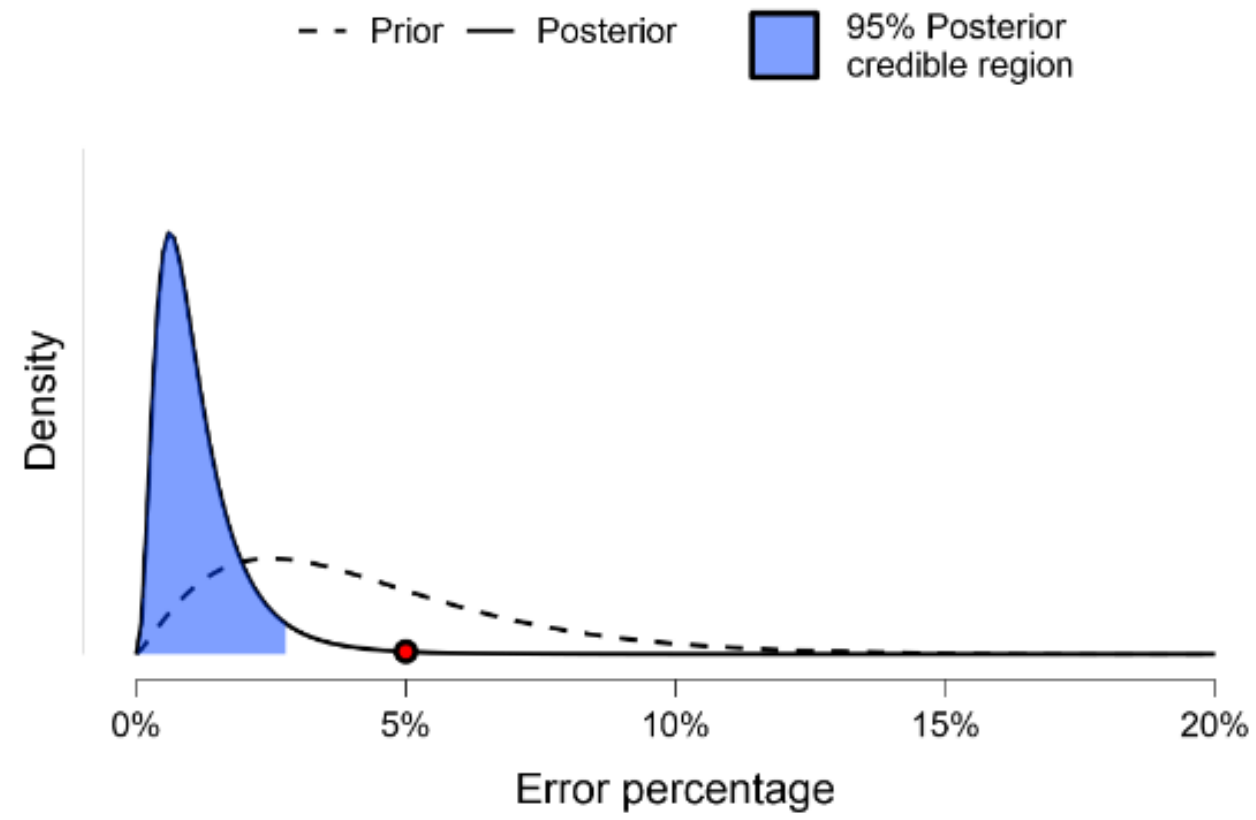

Figure 2. The prior and posterior probability distrubution (Cox and Snell) on the percentage of misstatement in the population. The red dot represents the specified materiality. If the credible area under the distribution surpasses this point, the estimate of the maximum misstatement exceeds the materiality.

\section{Conclusion}

To approve these data, a $95 \%$ upper credible bound on the population proportion of errors should be determined to be lower than materiality, in this case $\$ 70161$. For the current data, the credible bound is lower than materiality. The conclusion for these data is that the data contain no material misstatement.

Figure 10: Snapshot of the output screen of JfA's Bayesian evaluation stage containing the evaluation summary table and an annotation explaining the evaluation process. Below the evaluation summary table, the evaluation prior and posterior plot is displayed with a figure caption that explains the inference process. At the bottom, the correct conclusion is explained. 


\begin{abstract}
APPENDIX A
From a Bayesian point of view, Laura's assessment of the control risk can be incorporated in the prior distribution. For illustrative purposes, please consider the following example in which Laura wants to do a Bayesian audit and she wants to incorporate her assessment about the control risk into the prior.

Firstly, she needs to define the functional form of the prior distribution. She assumes the commonly used $\operatorname{Beta}(\alpha, \beta)$ prior distribution on $\theta$, the percentage of misstatement in the population (Stewart 2013). She assumes the likelihood for a sample size $n$, in which $k$ errors are found, to be $\operatorname{binomial}(n, k)$. Following Equation 2, this results in a $\operatorname{Beta}(\alpha+k, \beta+n-$ $k$ ) posterior distribution. Interestingly, a $\operatorname{Beta}(\alpha, \beta)$ prior can be interpreted as incorporating the information contained in a prior sample of size $\beta+\alpha-2$, in which $\alpha-1$ errors are assumed to be found.
\end{abstract}

Secondly, she needs to define what her expected error in the sample is. Based on last year's results, Laura expects to observe at most 2.5 percent misstatements in her sample.

Thirdly, she needs to define what kind of statement she wants to make after the audit is done. In BuildIt's case, Laura wants to be able to make the statement that when she finds at most 2.5 percent misstatements in the sample, she can infer with 95 percent confidence that the maximum percentage of misstatement in the population is below five percent (the materiality). The required sample size for this statement is determined as the lowest integer $n$, such that when 2.5 percent errors $(k=0.025 \times n)$ are observed, the upper credible bound of the posterior distribution is lower than five percent. When the form of the posterior, the materiality, and the expected error are known, this sample size is solely dependent on Laura's desired confidence level $\left(1-\alpha_{0}\right)$ and the prior parameters $\alpha$ and $\beta$, see Equation 4.

$$
n=P_{1-\alpha_{0}} \operatorname{Beta}(\alpha+0.025 n, \beta+n-0.025 n)<0.05
$$


When Laura has defined her assumptions about the form of the prior, her expected error, and her desired statement, she can incorporate the information from her assessment of the control risk in the prior distribution by setting the $\alpha$ and $\beta$ parameters of the prior distribution. Remember that these parameters can be interpreted as coming from a prior sample of size $\beta+\alpha-2$, in which $\alpha-1$ errors are found, and that increasing the detection risk results in a reduction of Laura's required sample. Hence, she sets the $\alpha$ and $\beta$ parameters by quantifying the effect that her adjustment in control risk has on her required sample. Therefore, she first calculates the sample size that is required for her statement when the control risk is set to high $\left(\alpha_{0}=0.05\right)$. When this risk assessment is high, the prior distribution is assumed to contain no information about $\theta$. The prior distribution that corresponds to this assumption is the $\operatorname{Beta}(1,1)$ distribution, as it assumes all values of $\theta$ to be equally likely to occur. The parameters of this prior distribution can be interpreted as coming from a sample of $\beta+\alpha-2=0$ transactions that contained $\alpha-1=0$ errors, and therefore the prior distribution assumes no prior information. Combining the $\operatorname{Beta}(1,1)$ prior with the binomial $(220,5.5)$ likelihood of an expected sample with 2.5 percent errors yields the $\operatorname{Beta}(6.5,215.5)$ distribution as a posterior distribution whose 95 percent $\left(1-\alpha_{0}\right)$ upper credible bound is below the materiality, see Equation 5 .

$$
P_{95} \operatorname{Beta}(1+0.025 \times 220,1+220-0.025 \times 220)<0.05
$$

This implies that when both risk assessments are set to high, a sample of 220 transactions in which at most 5.5 errors are found allows Laura to infer with 95 percent confidence that the maximum percentage of misstatement in the population is below five percent.

However, Laura asserted the control risk to be medium instead of high, which increased the detection risk from $\alpha_{0}=5$ percent to $\alpha_{1}=8.33$ percent. Combining the 
$\operatorname{Beta}(1,1)$ prior distribution with the binomial $(169,4.225)$ likelihood of an expected sample with 2.5 percent error yields the Beta $(5.225,165.775)$ as a posterior distribution whose 91.66 percent $\left(1-\alpha_{1}\right)$ upper confidence bound lies below the materiality, see Equation 6 .

$$
P_{91.66} \operatorname{Beta}(1+0.025 \times 169,1+169-0.025 \times 169)<0.05
$$

The prior information contained in the adjustment of the control risk from high to medium is captured in the reduction of the required sample, which is expressed through the reduction in the parameters of the first and second posterior distribution (de Swart, Wille, and Majoor 2013). Therefore, the prior distribution that corresponds to Laura's assessment of the control risk is the Beta(2.275.50.725) distribution. This implies that when the control risk is set to medium, a sample of 169 transactions in which at most 4.225 errors are found allows Laura to infer with 95 percent confidence that the maximum percentage of misstatement in the population is below five percent. 